\title{
Protective Effect of Valsartan on Podocyte Injury in Rats with Diabetic Nephropathy
}

\author{
Qingfen Wang ${ }^{1}$, , Rui $\mathrm{Li}^{1}$, Weiwei $\mathrm{Li}^{1}$, Lei Wang ${ }^{2}$ \\ ${ }^{1}$ Department of Nephrology, Binzhou People's Hospital, Binzhou, China \\ ${ }^{2}$ Department of Urology, Binzhou People's Hospital, Binzhou, China
}

Email address:

QingfenWangSDBZ@163.com (Qingfen Wang)

*Corresponding author

\section{To cite this article:}

Qingfen Wang, Rui Li, Weiwei Li, Lei Wang. Protective Effect of Valsartan on Podocyte Injury in Rats with Diabetic Nephropathy. American Journal of Life Sciences. Vol. 6, No. 3, 2018, pp. 47-51. doi: 10.11648/j.ajls.20180603.12

Received: November 28, 2018; Accepted: December 5, 2018; Published: December 6, 2018

\begin{abstract}
To investigate the mechanism of valsartan protecting podocytes and inhibiting renal injury in diabetic rats. The rat model of diabetic nephropathy was induced by combination of valsartan and high-sugar and high-fat diet. The urinary protein content, renal index, inflammatory and antioxidant indexes in the kidney, renal pathological changes and podocyte holes were investigated. Membrane WT1 and P-Cadherin protein expression levels. Compared with the model group, the 24h urine protein content of valsartan was significantly decreased $(\mathrm{P}<0.05)$. Valsartan significantly inhibited the body weight of the model group $(\mathrm{P}<0.01)$, and significantly inhibited The increase of renal index $(\mathrm{P}<0.05)$; the high and middle doses of valsartan could significantly reduce the levels of IL- $\beta$, TNF- $\alpha$ and IL-6 in rat kidney $(\mathrm{P}<0.05-0.01)$. The valsartan high and middle dose groups significantly reduced MDA content in rat kidney $(\mathrm{P}<0.05)$, and significantly increased SOD activity $(\mathrm{P}<0.05)$. HE and PAS staining showed that valsartan was used in model group rats. The pathological changes were alleviated, and the glomerular morphology returned to normal. The protein expression of WT1 and P-Cadherin in the kidney of DN rats by Western blot showed that P- in the kidney tissue of valsartan rats. The expression levels of Cadherin and WT1 protein were significantly increased $(\mathrm{P}<0.05)$. Valsartan can regulate the expression of podocyte membrane proteins WT1 and P-Cadherin to protect podocytes, and then repair renal function and anti-diabetic nephropathy.
\end{abstract}

Keywords: Valsartan, Diabetic Nephropathy, Podocyte, P-Cadherin, WT1 Protein

\section{Introduction}

Diabetic nephropathy (DN) is the most common chronic microangiopathy in diabetes, and its early pathological manifestations are mesangial cell matrix accumulation and glomerular basement membrane thickening [1]. Currently, clinical treatment of $\mathrm{DN}$ is mainly through stabilizing blood sugar and blood pressure, regulating dyslipidemia, and using angiotensin receptor antagonists (ARBs) or angiotensin converting enzyme inhibitors (ACEI), etc. [2], such as irbe Shatan. Studies have shown that irbesartan can block early TLR4 signaling pathway and treat early diabetic nephropathy, and can also be used in combination with other western medicines or proprietary Chinese medicines to treat early diabetic nephropathy [3]. However, it is worth noting the adverse effects of irbesartan, such as allergic purpura, eczema, hyperkalemia, severe hypertension, lymph nodes and breast pain [4].

\section{Materials and Methods}

\subsection{Experimental Animals}

SPF grade SD rats, male, body weight $(220 \pm 20) \mathrm{g}$, provided by the Experimental Animal Center of Shandong University, certificate number: SYXK-2013-0054. The animal experiments carried out in this study were approved by the Animal Ethics Committee. Rats were housed in cages and kept at room temperature $23^{\circ} \mathrm{C}$, humidity $40 \%, 12 \mathrm{~h}$ day and night, free drinking water, standard normal feed.

\subsection{Main Instruments, Reagents and Drugs}

Streptozotocin (Sigma); rabbit anti-rat P-Cadherin 
polyclonal antibody (Abeam, USA); rabbit anti-rat WT1 polyclonal antibody (Beijing Boaosen Bio, China); TRIzol Reagent (Life Technologies, Inc.), USA); MMLV Reverse Transcription Kit (Invitrogen, USA). Johnson \& Johnson Steady Blood Glucose Tester (Johnson \& Johnson China, China); Microplate Reader (Prang Medical Co., Ltd., China); Analytical Balance (Sartorius, Germany); automatic sample rapid grinding instrument (China Shanghai Netcom Technology Co., Ltd., China); desktop frozen micro-speed centrifuge (Thermo Micro7R, USA); ultraviolet spectrophotometer (Therm, USA); ordinary PCR instrument (Thermo TCA5020, USA); Fluorescence Quantitative PCR Cycler (ABI 7500, USA); Gel Imaging System (UVP BioDoct220, United States); pipette (Thermo Finnpipette, United States).

\subsection{Rat Model Preparation and Grouping}

SD rats were fed for 1 week. After adjusting to the environment, urine protein and urine glucose were negative. A small dose of high-fat diet were combined with DN rat model. 48 rats were fed with high-sugar and high-fat diet for 4 weeks. After fasting for 12 hours, $1 \%$ valsartan was injected intraperitoneally. Blood glucose was measured by venous blood sampling, and the blood glucose level $\geq 16.7 \mathrm{mmol} / \mathrm{L}$ was a successful model of diabetes. Rats who died and whose blood glucose was not up to standard were removed during the modeling process. Diabetic rats were randomly divided into a model group (MOD), a positive drug (irbesartan, IRB) group, and a valsartan (high, medium, and low dose) group, with 8 rats in each group. The eubesartan tablets $(0.15 \mathrm{~g} /$ tablet $)$ were administered at a dose of 15 $\mathrm{mg} /(\mathrm{kg} \cdot \mathrm{d})$, while the rats in the normal group and the model group were given corresponding volumes of distilled water, and each group was continuously intragastrically administered for 16 weeks.

\subsection{Cytokine Detection}

Blood was taken from the tail vein of rats at $4,8,12$, and 16 weeks after the intervention of valsartan, and blood glucose levels were measured. The total amount of $24 \mathrm{~h}$ urine protein in each group was determined according to the kit instructions and its steps to determine the total amount of $24 \mathrm{~h}$ urine protein. After the rats were sacrificed by cervical dislocation, the bilateral renal tissues were removed, and the blood on the kidney surface was blotted with a filter paper, and the mass was weighed. Kidney index $=$ sum of bilateral kidney mass / body mass x $100 \%$.

\subsection{Renal Pathology Examination}

Take renal cortex, formaldehyde (10\%) fixed for $24 \mathrm{~h}$, rinse with running water, dehydrate each time with $70 \%, 80 \%, 90 \%$, $95 \%, 100 \%$ ethanol gradient for $30 \mathrm{~min}$, xylene transparent, dip wax, paraffin embedded, slice The thickness is about $2 \mu \mathrm{m}$. After HE and PAS staining, the pathological changes of renal tissue were observed under light microscope.

\subsection{Detection of Inflammatory Factors in the Kidney}

Rats in each group were sacrificed by cervical dislocation 1 $\mathrm{h}$ after the last administration. The bilateral kidney tissues were removed. After homogenization, the cells were homogenized at $12000 \mathrm{r} / \mathrm{min}$, centrifuged for $15 \mathrm{~min}$, and the supernatant was taken for testing. The inflammatory factors (IL-1, IL-6 and TNF- $\alpha$ ), SOD activity and MDA content in all rat kidneys were detected by ELISA kit. The specific procedures were carried out according to the instructions on the kit.

\subsection{Detection of WT1 and P-Cadherin Protein Expression}

$60 \mathrm{mg}$ of rat kidney tissue was taken, RNA was extracted using protein lysate RIPA, and protein concentration was measured using a Bradford protein quantification kit. Take 30 $\mu \mathrm{g}$ of each sample, add the total protein sample and the protein gel electrophoresis loading buffer solution, mix well, denature at $95^{\circ} \mathrm{C}$ for $10 \mathrm{~min}$, ice bath, set aside. The sample $(30 \mu \mathrm{g})$ was slowly added to the gel hole, and the electrophoresis was under a steady state with a voltage of $80 \mathrm{~V}$. The sample was passed through a concentrated gel and a separating gel (voltage $8 \mathrm{~V} / \mathrm{cm}$ ). The dye was electrophoresed to the appropriate position of the separation gel, and the membrane was transferred on ice. The PVDF membrane with protein was blocked with $5 \%$ skim milk powder at $4{ }^{\circ} \mathrm{C}$, and the rabbit anti-rat P-Cadherin primary antibody $(1: 1 \mathrm{~kg})$ was added. Rat WT1 primary antibody $(1: 300)$, washed with TBST, the washed primary antibody reaction membrane was placed in the secondary antibody working solution $(1: 5000)$, at room temperature, protected from light, slowly shaken, after 60 minutes, the membrane was washed., TMB color development, exposure washing, the relative content of the target protein is represented by the gray ratio of the target protein / $\beta$-actin.

\subsection{Statistical Methods}

The experimental data were expressed by $\mathrm{x} \pm \mathrm{s}$, and one-way analysis of variance was performed using SPSS 19.0 statistical analysis software. $\mathrm{P}<0.05$ was used to indicate statistical difference.

\section{Results}

\subsection{Effects of Valsartan on Body Weight, Kidney Mass and Renal Index in Diabetic Rats}

After 16 weeks of modeling, the body mass and kidney mass of each group were weighed, and the renal index was calculated. The results showed that compared with the normal group, the body weight of the model group was significantly lower $(\mathrm{P}<0.01)$ and the renal index was significantly increased $(\mathrm{P}<0.01)$. After the intervention of valsartan, the valsartan high and middle dose groups were all The body mass of the model group was significantly inhibited $(\mathrm{P}<0.05-0.01)$, and the renal index of rats with diabetic nephropathy was significantly improved $(\mathrm{P}<0.05-0.01)$. The results are shown in figure 1. 

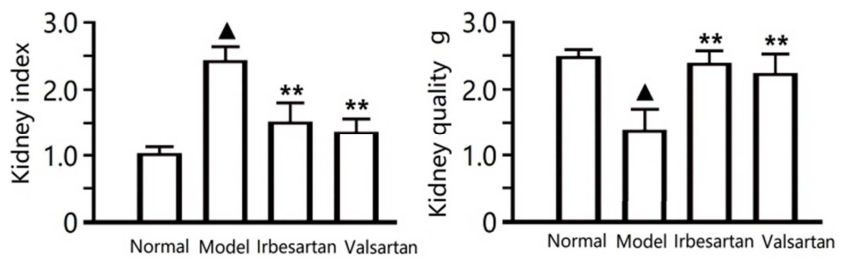

Figure 1. Effects of valsartan on body weight, kidney mass and renal index in diabetic rats.

\subsection{Renal Pathology Examination}

The results of light microscopy showed that the normal $(\mathrm{CON})$ group had normal renal morphology, clear structure, regular globular shape, no expansion of the capillary lumen, and tightly arranged tubules. Renal tubular epithelial cell morphology Normal, glomerular no atrophy or hypertrophy, no thickening of the basement membrane, no hyperplasia of the mesangium, the result is labeled as "-"; compared with the normal group, the volume of glomerulus of the model group (MOD) rats increased, The capillary basement membrane was obviously thickened, the mesangial matrix hyperplasia was obvious, and the renal tubular epithelial cells were vacuolated, and the protein cast type was observed. The result was labeled as " ++++ ", and the pathological changes of the valsartan group were all relieved to some extent. The glomerular morphology was basically normal. The basement membrane was partially thickened and the mesangial membrane was slightly hyperplasia. Only a small number of renal tubular epithelial cells were vacuolated, and the degree of lesion was significantly lighter than that of the model group. The valsartan-L group results were marked. For "+++", the valsartan-M group results were labeled "++" and the valsartan-H group results were labeled "+". The results are shown in figure 2 .

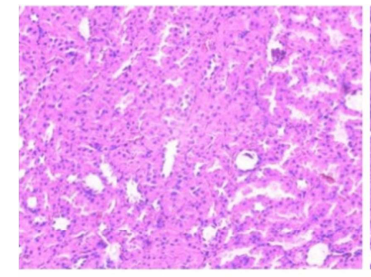

Normal

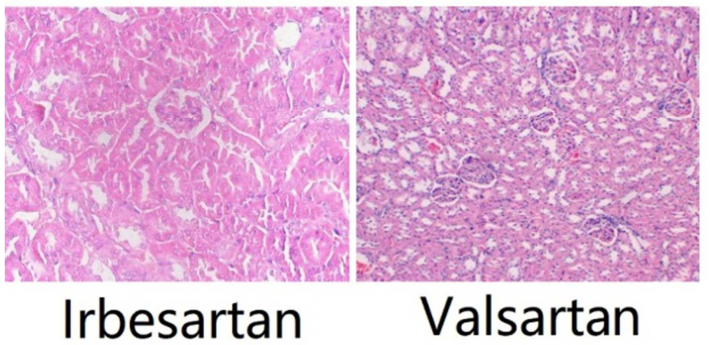

Figure 2. Renal pathology examination.

\subsection{PAS Staining}

The ratio of the area stained positive to the area of each tangential glomerulus was calculated using the Leica Q500MC analysis software. Results Compared with the normal group, the ratio of the positive area of renal tissue staining to the glomerular area of the model group was significantly increased. Compared with the model group, the irbesartan group and the valsartan group all reduced the early stage of diabetic nephropathy. The positive area of rat kidney tissue staining $(\mathrm{P}<0.05)$ improved glomerular volume, basement membrane thickening, and mesangial proliferation. The result is shown in figure 3 .

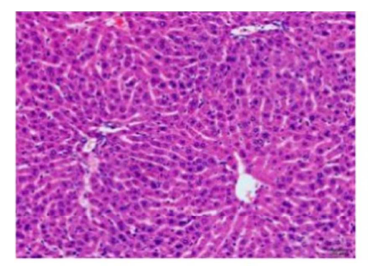

Normal

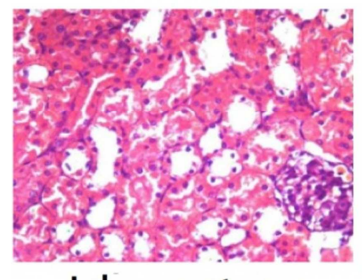

Irbesartan

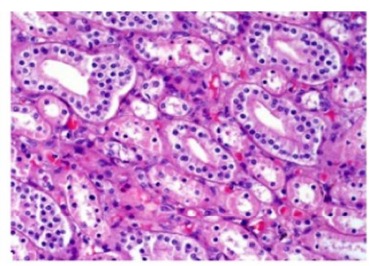

Model

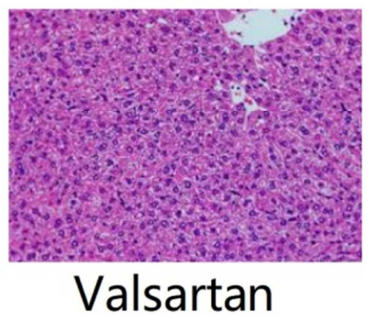

Figure 3. PAS staining.

\subsection{Detection of Inflammatory Factors and Antioxidant Markers in the Kidney}

Compared with the normal group, the concentrations of IL- $\beta$, TNF- $\alpha$ and IL- 6 in the kidney of the model group were significantly increased $(\mathrm{P}<0.01)$. Compared with the model group, valsartan significantly decreased IL in the kidney of rats. The concentrations of $-\beta$, TNF- $\alpha$ and IL- 6 were significant $(\mathrm{P}<0.05)$. The results of antioxidant index showed that the SOD activity in the kidney of the model group was significantly decreased $(\mathrm{P}<0.01)$, the concentration of MDA was significantly increased $(\mathrm{P}<0.01)$, and the valsartan high and middle dose groups were significantly reduced in the model group. MDA concentration in rat kidney $(\mathrm{P}<0.05)$, valsartan high, medium and low dose groups significantly increased SOD activity $(\mathrm{P}<0.05)$. The result is shown in figure 4 .
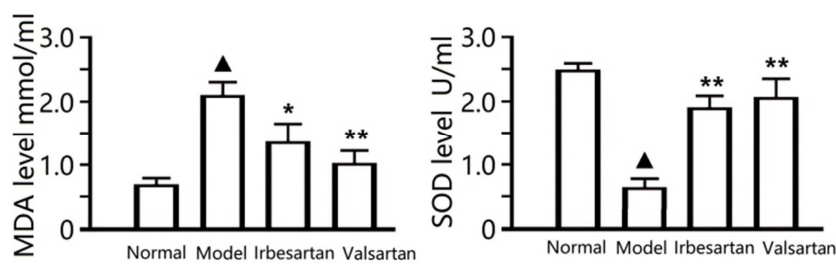

Figure 4. Detection of inflammatory factors and antioxidant markers in the kidney.

\subsection{WT1 and P-Cadherin Protein Expression Detection}

Compared with the normal group, the expression of P-Cadherin and WT1 protein in the renal tissue of the model group decreased $(\mathrm{P}<0.01)$; compared with the model group, the irbesartan group and the valsartan high and middle dose 
group rats The expressions of $\mathrm{P}-\mathrm{Cadherin}$ and $\mathrm{WT} 1$ protein in renal tissues were increased $(\mathrm{P}<0.01)$. Compared with the model group, the expression of P-Cadherin and WT1 protein in renal tissues of valsartan was significantly increased $(\mathrm{P}<0.05)$. There was no significant difference in the expression levels of the two proteins in the high dose group of valsartan compared with the irbesartan group. The results are shown in figure 5.
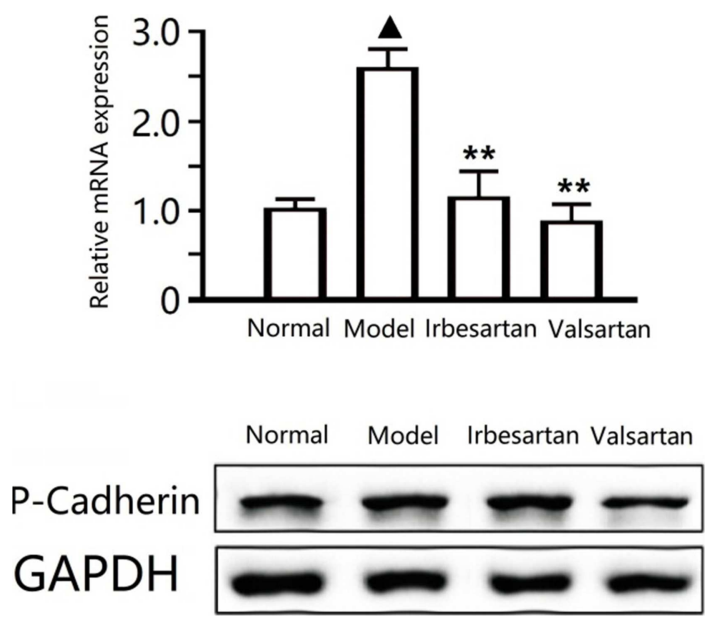

Figure 5. WT1 and P-Cadherin protein expression detection.

\section{Discussion}

This experimental study showed that valsartan had no significant difference with irbesartan in reducing the amount of urinary protein in DN rats [5], down-regulating the levels of inflammatory factors in the kidney, regulating MDA and SOD levels, and protecting podocytes $[6,7]$. Therefore, the author believes that valsartan has an irreplaceable role in the repair of diabetic nephropathy, and has a good development and application prospects. Podocytes are an important component of glomerular filtration function. The podocyte membrane is the last barrier of glomerular filtration and plays a regulatory role in the production of proteinuria [8, 9]. Nephrin-WT1-P-Cadherin complex is a key functional unit that constitutes a ruptured membrane and is an important condition for maintaining glomerular filtration function [10, 11]. Studies have shown that P-Cadherin is a structural protein of the podocyte membrane and concentrates on the tight junction between the foot processes, which binds the tight junction protein to the actin of the podocytes and acts as a scaffold $[12,13]$. WT1 is another important protein that maintains the function and structure of the podocyte membrane. It not only interacts with various pore-membrane proteins such as P-Cadherin, but also with cytoskeletal proteins and bridging proteins to maintain podocyte foot processes. Normal state $[14,15]$.

The production of proteinuria is mainly related to the glomerular filtration barrier, which is a highly differentiated epithelial cell attached to the glomerular basement membrane and is a major component of the glomerular filtration barrier. As the last barrier of glomerular filtration, it plays an important role in the occurrence of proteinuria [16]. The dysregulation, dysfunction and localization of WT1 protein can cause abnormal aggregation and distribution of podocyte cytoskeletal proteins, which are closely related to the occurrence and development of proteinuria and glomerular lesions [17]. WT1 knockout mice cause renal foot cell foot process defects, extensive fusion, large amounts of proteinuria, and can increase susceptibility to glomerular injury, leading to glomerular sclerosis. Defects in WT1 can also lead to fusion of the foot processes through deposition of the mesangial matrix, leading to proteinuria $[18,19]$.

\section{Consideration}

Diabetic nephropathy (DN) is the most important microvascular disease in patients, also leading to end-stage kidney. An important cause of the disease. Microalbuminuria is not only the main clinical indicator of DN injury, but also an independent risk of promoting glomerular sclerosis factor. Renal tissue local renin angiotensin system (RAS) activation, angiotensin II (AngII) activity increased, causing local hemodynamic changes in the kidney, especially glomerular capillaries increased internal pressure leads to increased urinary albumin excretion; valsartan belongs to the angiotensin receptor subtype (AT1) antagonist agent, which blocks the binding of AngII by blocking the AT1 receptor less, lower systemic blood pressure, improve hemodynamic changes, reduce white urine delays the occurrence and development of DN. In consideration, valsartan can reduce the amount of urine protein, repair the pathological damage of kidney to kidney and protect kidney function by up-regulating the expression of WT1P-Cadherin.

\section{References}

[1] Wadie W, El-Tanbouly DM. Vinpocetine mitigates proteinuria and podocytes injury in a rat model of diabetic nephropathy. European journal of pharmacology. 2017; 814: 187-95.

[2] Peng T, Chang X, Wang J, Zhen J, Yang X, Hu Z. Protective effects of tacrolimus on podocytes in early diabetic nephropathy in rats. Molecular medicine reports. 2017; 15 (5): 3172-8.

[3] Mukhi D, Nishad R, Menon RK, Pasupulati AK. Novel Actions of Growth Hormone in Podocytes: Implications for Diabetic Nephropathy. Frontiers in medicine. 2017; 4: 102.

[4] Liu Y, Zhang J, Wang Y, Zeng X. Apelin involved in progression of diabetic nephropathy by inhibiting autophagy in podocytes. Cell death \& disease. 2017; 8 (8): e3006.

[5] Huang SS, Ding DF, Chen S, Dong CL, Ye XL, Yuan YG, et al. Resveratrol protects podocytes against apoptosis via stimulation of autophagy in a mouse model of diabetic nephropathy. Scientific reports. 2017; 7: 45692.

[6] Hsu YH, Li HH, Sung JM, Chen WY, Hou YC, Weng YH, et al. Interleukin-20 targets podocytes and is upregulated in experimental murine diabetic nephropathy. Experimental \& molecular medicine. 2017; 49 (3): e310. 
[7] Guo Y, Song Z, Zhou M, Yang Y, Zhao Y, Liu B, et al. Infiltrating macrophages in diabetic nephropathy promote podocytes apoptosis via TNF-alpha-ROS-p38MAPK pathway. Oncotarget. 2017; 8 (32): 53276-87.

[8] Zhang H, Luo W, Sun Y, Qiao Y, Zhang L, Zhao Z, et al. Wnt/beta-Catenin Signaling Mediated-UCH-L1 Expression in Podocytes of Diabetic Nephropathy. International journal of molecular sciences. 2016; 17 (9).

[9] Tharaux PL, Huber TB. How Is Proteinuric Diabetic Nephropathy Caused by Disturbed Proteostasis and Autophagy in Podocytes? Diabetes. 2016; 65 (3): 539-41.

[10] Sawada K, Toyoda M, Kaneyama N, Shiraiwa S, Moriya H, Miyatake H, et al. Upregulation of alpha3beta1-Integrin in Podocytes in Early-Stage Diabetic Nephropathy. Journal of diabetes research. 2016; 2016: 9265074.

[11] Liu F, Zong M, Wen X, Li X, Wang J, Wang Y, et al. Silencing of Histone Deacetylase 9 Expression in Podocytes Attenuates Kidney Injury in Diabetic Nephropathy. Scientific reports. 2016; 6: 33676 .

[12] Ji ZZ, Xu YC. Melatonin protects podocytes from angiotensin II-induced injury in an in vitro diabetic nephropathy model. Molecular medicine reports. 2016; 14 (1): 920-6.

[13] He Y, Zhang M, Wu Y, Jiang H, Fu H, Cai Y, et al. Aberrant activation of Notch-1 signaling inhibits podocyte restoration after islet transplantation in a rat model of diabetic nephropathy. Cell death \& disease. 2018; 9 (10): 950.
[14] Zhang Y, Chen X, Yuan L, Wu J, Guo N, Liu J. Down-regulation of IRAK1 attenuates podocyte apoptosis in diabetic nephropathy through PI3K/Akt signaling pathway. Biochemical and biophysical research communications. 2018; 506 (3): 529-35.

[15] Lei X, Zhang L, Li Z, Ren J. Astragaloside IV/lncRNA-TUG1/TRAF5 signaling pathway participates in podocyte apoptosis of diabetic nephropathy rats. Drug design, development and therapy. 2018; 12: 2785-93.

[16] Wang Y, Li H, Song SP. beta-Arrestin 1/2 Aggravates Podocyte Apoptosis of Diabetic Nephropathy via Wnt/beta-Catenin Pathway. Medical science monitor: international medical journal of experimental and clinical research. 2018; 24: 1724-32.

[17] Pan Y, Jiang S, Hou Q, Qiu D, Shi J, Wang L, et al. Dissection of Glomerular Transcriptional Profile in Patients With Diabetic Nephropathy: SRGAP2a Protects Podocyte Structure and Function. Diabetes. 2018; 67 (4): 717-30.

[18] Wang XB, Zhu H, Song W, Su JH. Gremlin Regulates Podocyte Apoptosis via Transforming Growth Factor-beta (TGF-beta) Pathway in Diabetic Nephropathy. Medical science monitor: international medical journal of experimental and clinical research. 2018; 24: 183-9.

[19] Lv Z, Hu M, Fan M, Li X, Lin J, Zhen J, et al. Podocyte-specific Rac1 deficiency ameliorates podocyte damage and proteinuria in STZ-induced diabetic nephropathy in mice. Cell death \& disease. 2018; 9 (3): 342. 